\title{
Distinct roles for medial temporal lobe structures in memory for objects and their locations
}

\author{
Elizabeth A. Buffalo, ${ }^{1,3}$ Patrick S.F. Bellgowan, ${ }^{2}$ and Alex Martin ${ }^{2}$ \\ ${ }^{1}$ Department of Neurology, Emory University School of Medicine, and the Yerkes National Primate Research Center, Atlanta, \\ Georgia 30329, USA; ${ }^{2}$ Laboratory of Brain and Cognition, National Institute of Mental Health, Bethesda, Maryland 20892, USA
}

\begin{abstract}
The ability to learn and retain novel information depends on a system of structures in the medial temporal lobe $(\mathrm{MTL})$ including the hippocampus and the surrounding entorhinal, perirhinal, and parahippocampal cortices. Damage to these structures produces profound memory deficits; however, the unique contribution to memory of each of these structures remains unclear. Here we have used functional magnetic resonance imaging (fMRI) to determine whether the perirhinal and parahippocampal cortices show differential memory-related activity. Based on the distinct patterns of cortical input to these two areas, we reasoned that these structures might show differential activity for spatial and object recognition memory. In each of 11 subjects, we found that the perirhinal cortex was active during both spatial and object memory encoding, while the anterior parahippocampal cortex was active only during spatial encoding. These data support the idea that MTL structures make distinct contributions to recognition memory performance.
\end{abstract}

Long-term memory encoding and retrieval critically depend on a system of interconnected structures in the medial temporal lobe (MTL), including the hippocampus and the surrounding entorhinal, perirhinal, and parahippocampal cortices. Neuroimaging studies have shown that MTL structures are activated during encoding (Brewer et al. 1998; Wagner et al. 1998; Reber et al. 2002; Pihlajamaki et al. 2003), delay (Stark and Squire 2000; Ranganath and D'Esposito 2001; Rombouts et al. 2001), and retrieval (Stark and Squire 2000; Rombouts et al. 2001). However, it is unclear whether and how these structures make distinct contributions to memory.

There are strong anatomical reasons for supposing that MTL cortices have distinct memory functions in terms of modality specificity (Suzuki and Amaral 1994a). The perirhinal cortex receives a larger percentage of its cortical input from higher-order visual association areas than does the parahippocampal cortex. In contrast, the parahippocampal cortex receives a larger percentage of its cortical input from spatial areas. Furthermore, there is an asymmetry in the connectivity between these areas: The perirhinal cortex provides a relatively weak projection to the parahippocampal cortex, while the parahippocampal cortex projects strongly to the perirhinal cortex. Taken together, these data suggest that the parahippocampal cortex might be more important for spatial memory and the perirhinal cortex might be more important for object memory. However, these data also raise the alternative possibility that the perirhinal cortex is similarly involved in spatial and object memory because of the large amount of spatial information that the perirhinal cortex receives via the parahippocampal cortex (Fig. 1).

Behavioral studies of the effects of medial temporal lobe lesions in monkeys and humans have raised the possibility of such a dissociation between the perirhinal cortex and the parahippocampal cortex (Mahut 1971; Jones and Mishkin 1972; Bohbot et al. 1998; Murray et al. 1998; Malkova and Mishkin 2003). However, because the roles of these cortices have been investigated in separate experiments or with combined lesions

\section{${ }^{3}$ Corresponding author.}

E-mail elizabeth.buffalo@emory.edu; fax (404) 727-9294.

Article published online before print. Article and publication date are at http:// www.learnmem.org/cgi/doi/10.1101/lm.251906. including other MTL structures, it has been difficult to assess directly the relative importance of each structure's contribution to memory.

Functional neuroimaging techniques are particularly well suited for addressing this question because they allow for a within-subject comparison of cortical activations as well as an investigation of possible functional subdivisions within defined cytoarchitectonic regions. To date, very few functional imaging studies of recognition memory have examined possible distinctions between medial temporal lobe structures (Davachi et al. 2003; Duzel et al. 2003; Pihlajamaki et al. 2003, 2004; Kohler et al. 2005; Sommer et al. 2005), and no direct comparisons have been made between the anatomically defined perirhinal and parahippocampal cortices while subjects explicitly perform a memory task across modalities. Here, we compared the activation patterns of the perirhinal cortex and the parahippocampal cortex while subjects performed tasks of visual object and spatial recognition memory. The extent of the perirhinal and parahippocampal cortex was defined using anatomical landmarks, for each subject individually. The results demonstrate that these structures make distinct contributions to recognition memory. The perirhinal cortex was active during the encoding of both objects and locations. In contrast, at the border between the perirhinal and parahippocampal cortices, a region in the anterior parahippocampal cortex was active only during the encoding of locations. Finally, posterior parahippocampal cortex was active during the encoding of both tasks.

\section{Results}

\section{Recognition memory performance}

A description of the tasks is given in Figure 2. In alternating blocks of trials, subjects performed an object or spatial recognition memory task. Item recognition (percent correct) across all subjects during the functional scans did not differ for the two tasks $\left(F_{1,10}<1 ; P>0.10\right)$. The average performance was $77 \%$ correctly recognized items for the object task and $76 \%$ for the spatial task. Performance as measured by d-prime also did not differ for the two tasks $\left(F_{1,10}<1 ; P>0.10\right)$. The average d-prime rate was 2.22 for the Object task and 2.16 for the Spatial task. Subjects' reaction time in the two tasks was also not significantly different 


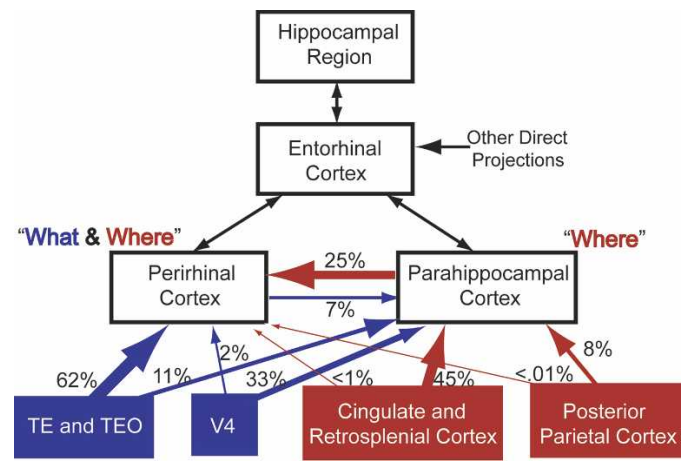

Figure 1. Cortical input to the medial temporal lobe. A schematic diagram showing the percentage of cortical input from unimodal and polymodal areas to the perirhinal and parahippocampal cortices in the medial temporal lobe (black boxes). The percentages of cortical input shown on this schematic are from Suzuki and Amaral (1994a). These data suggest that the parahippocampal cortex might be more important for spatial memory (red lines and boxes), while the perirhinal cortex might be more important for object memory (blue lines and boxes). Additionally, these data raise the possibility that the perirhinal cortex is involved in both spatial and object memory because of the large amount of spatial information it receives via the parahippocampal cortex. The thickness of the lines approximately represents the relative percentages of cortical input.

$\left(F_{1,10}=2.13 ; P>0.05\right)$. The median reaction time across subjects was $1211 \mathrm{msec}$ for the Object task and $1096 \mathrm{msec}$ for the Spatial task. Accordingly, any task-dependent activation differences across brain regions could not be attributed to differences in task difficulty. With whole-brain scanning, in a pilot subject, clear differences were seen in ventral and dorsal stream visual regions, suggesting that subjects used differential processes to solve the two tasks (Fig. 3).

\section{fMRI task effects}

Using anatomically defined regions of interest, we compared activity in the perirhinal and parahippocampal cortices during performance of the Object and Spatial recognition memory tasks. Figure 4 shows the location of significant task-specific fMRI activity during encoding across the group of 11 subjects. Activity during encoding that was stronger for the Object than the Spatial task was seen throughout the ventral visual stream. In contrast, a region in the anterior parahippocampal cortex bilaterally was selectively active when subjects were encoding locations. The perirhinal cortex was active during encoding of both objects and locations (see Fig. 5), and there was no task-specific encoding activity in the perirhinal cortex.

Figures 5 and 6 show the location of the activity seen in the group analysis in the perirhinal and parahippocampal cortex for each subject individually. Across the group and in each of the individual subjects, there was significant activity in the perirhinal cortex during encoding that was not different between the two tasks (Fig. 5). Encoding activity in the perirhinal cortex was located in both banks of the collateral sulcus, and there was bilateral activation in seven subjects.

The anterior parahippocampal cortex was significantly more active during the Spatial task than during the Object task, and this differential activity was seen in each of the 11 subjects (Fig. 6). The activation in the anterior parahippocampal cortex was located in both banks of the collateral sulcus, immediately posterior to the perirhinal-parahippocampal border, and was predominately lateralized to the right hemisphere. However, seven subjects showed this activation bilaterally. This spatial encoding activation did not extend throughout the entire rostro-caudal extent of the parahippocampal cortex, but was limited to the most rostral portion of the parahippocampal cortex $(\sim 8-12 \mathrm{~mm}$; two to three functional coronal slices).

Figure 7 shows the averaged perirhinal, anterior parahippocampal, and posterior parahippocampal cortex activation for all subjects during the encoding phase of both tasks. There was a significant difference in activity during the two tasks only in the anterior parahippocampal cortex $(P<0.001)$, where there was greater activity during spatial encoding than during object encoding. There was a trend for the perirhinal cortex to be more active during object encoding, but this was not significant $(P=0.08)$.

We concentrated our analysis on the encoding phase of the tasks because activity during the recognition phase could reflect either recognition of the repeated stimuli or encoding of the novel stimuli. However, we found similar results across regions during the recognition phase. The perirhinal cortex and the posterior parahippocampal cortex were active during the recognition phase for both the Spatial and Object tasks $(P<0.01)$. Unlike encoding, the anterior parahippocampal cortex was not active during the recognition phase of either task $(P>0.10)$. None of the regions showed significant activity above baseline during the delay period $(P>0.10)$.

\section{Discussion}

The results from the present experiment suggest that components of the MTL make distinct contributions to memory performance. We found differences in activity in the perirhinal and parahippocampal cortices for visual object and spatial recognition memory. A region in the anterior part of the parahippocampal cortex was active only when subjects were encoding stimuli in the Spatial memory task. In contrast, the perirhinal cortex was similarly active when subjects performed the Object and the Spatial memory tasks. The posterior parahippocampal cortex was active during both object and spatial encoding.

It has been previously suggested that the perirhinal and parahippocampal cortices may play distinct roles in visual object and spatial memory, respectively (Suzuki and Amaral 1994a). The data from the present experiment provide the first direct evidence that such a functional distinction exists. One unexpected finding of the present experiment was that the spatial activation in the parahippocampal cortex was restricted to the anterior portion. To our knowledge, there are no anatomical studies of the MTL that have analyzed tracer injections restricted to this anterior region that might suggest an anterior-posterior gradient in the input of spatial information to the parahippocampal cortex.
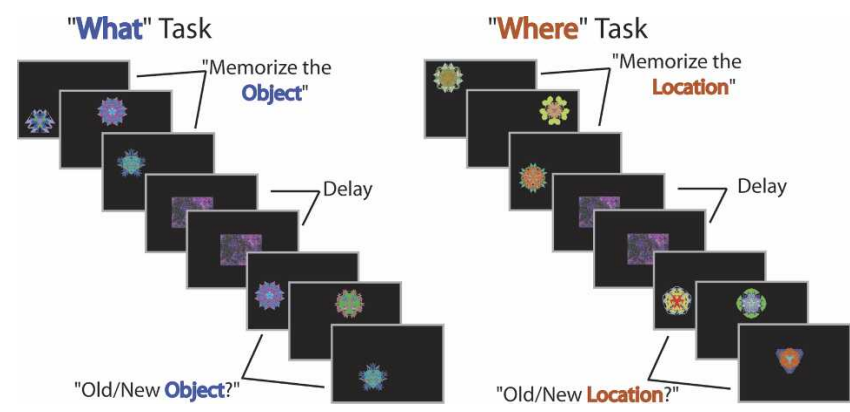

Figure 2. Task design. While being scanned, subjects were shown a series of six colored stimuli and were instructed to memorize the object (Object task) or memorize the location (Spatial task). During a delay, subjects viewed a scrambled image presented six times at the center of the screen. During the recognition phase, subjects were shown three old objects and three new objects (Object task) or objects in three old locations and three new locations (Spatial task). For each stimulus, subjects made an Old/New judgment by pressing buttons on a button box. 

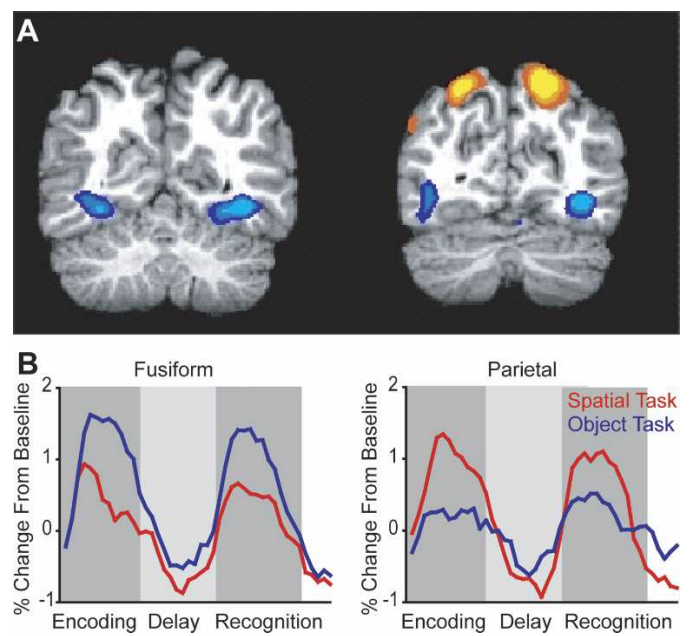

Figure 3. What versus where task activity. In an individual subject, the two tasks activated different areas in the visual processing pathway. $(A)$ Areas that showed greater activation during the Object task (cool colors) or during the Location task (warm colors). (B) The respective averaged time series for these activations.

The data from the present study raise this possibility, which would be an interesting topic for a future anatomical study.

Behavioral studies in monkeys and humans have reported spatial impairments resulting from lesions of the parahippocampal cortex (Jones and Mishkin 1972; Bohbot et al. 1998; Ploner et al. 2000; Malkova and Mishkin 2003). It is interesting to note, however, that in a recent study (Malkova and Mishkin 2003), one animal with a parahippocampal cortex lesion was unimpaired on a spatial memory task (object-place association), while the rest of the animals in this lesion group were impaired. Postmortem analysis of the lesion in the unimpaired animal revealed tissue sparing in the anterior parahippocampal cortex. Although this is just one animal, this finding is consistent with the present study and suggests that the anterior parahippocampal cortex might be critical for certain kinds of spatial memory.

In a recent study, Sommer et al. (2005) reported an area of activation in the right anterior medial temporal lobe that was correlated with memory for the location associated with an object. Using an event-related design, these authors correlated activity during encoding an object-location association with retrieval success when presented with either an object cue or a location cue. They found that activity in a small region in the right anterior MTL during encoding correlated exclusively with spatial encoding processes. That is, greater activity in this region during encoding predicted greater retrieval success with a location cue. The results in the present study support and extend this finding. Their anterior MTL activation overlaps exactly with the region in the anterior parahippocampal cortex in which we found greater activation during spatial encoding. In the present study, the use of precise anatomical landmarks allowed us to clearly identify this area that appears to be specialized for spatial memory.

Previous studies have suggested that there is a region in the posterior part of the parahippocampal gyrus specialized for the processing of spatial layouts in humans (Epstein et al. 1999; Bar and Aminoff 2003). In the present study, we found strong activity in the posterior parahippocampal cortex during encoding and recognition of both objects and locations. We found no evidence for specialized spatial processing in the posterior parahippocampal cortex. One important difference in the present study and previous studies was in the stimuli used in the spatial memory task. Epstein et al. (1999) and Bar and Aminoff (2003) used pic- tures of houses and objects in their familiar context, respectively, to activate the posterior parahippocampal cortex. Pihlajamaki et al. (2004) used different arrangements of familiar stimuli. Accordingly, these tasks measured responses to contextualized spatial images. In the present experiment, spatial memory consisted of remembering an exact location on the computer screen, out of a possible 16 , of stimulus presentation. That is, the stimuli to be remembered were simply spatial coordinates without any realworld object counterpart. Another aspect of this task was the lack of rehearseability of the items to be remembered. Subjects were debriefed after the scanning, and subjects reported that they sometimes tried to attach a verbal label to a stimulus location, but were not able to do this for more than one or two stimuli in a block. Additionally, not one subject was aware that the stimuli were presented on a $4 \times 4$ grid of 16 possible locations. It is therefore possible that the anterior parahippocampal cortex is important for this kind of abstract spatial memory, while the posterior parahippocampal cortex is involved in the processing of objects with contextualized spatial associations.

The present findings are in conflict with a recent study showing that the posterior parahippocampal cortex was preferentially activated while subjects viewed changes in the spatial arrangement of familiar stimuli (Pihlajamaki et al. 2004). A crucial difference between these studies is that in the present study, subjects were explicitly asked to remember the location of the stimuli in the spatial task. Pihlajamaki et al. (2004) asked subjects to look carefully at stimuli but did not ask them to remember the stimuli for later testing. It is possible that these task differences led to differential activation of these regions.

The present findings suggest that the perirhinal cortex is equally involved during the encoding of objects and locations. This is somewhat surprising, given that the perirhinal cortex receives the majority of its cortical input from higher-order visual object association areas (see Fig. 1). However, the perirhinal cortex receives strong input from the parahippocampal cortex, which could be the source of spatial information to this area. The perirhinal cortex has been shown to be critical for both visual and tactile recognition memory performance (Buffalo et al. 1999). The present data suggest that the perirhinal cortex is similarly involved in spatial recognition memory.

An alternative possibility is that because spatial locations were repeated, the activity seen in the perirhinal cortex during spatial encoding actually reflected the retrieval of objects previously associated with a given location. Although this is important to consider, we believe that the previous interpretation better fits our data for several reasons. First, as shown in Figure 3, in a pilot subject, we found a clear dissociation in activity between ventral occipitotemporal and parietal areas for the Object and

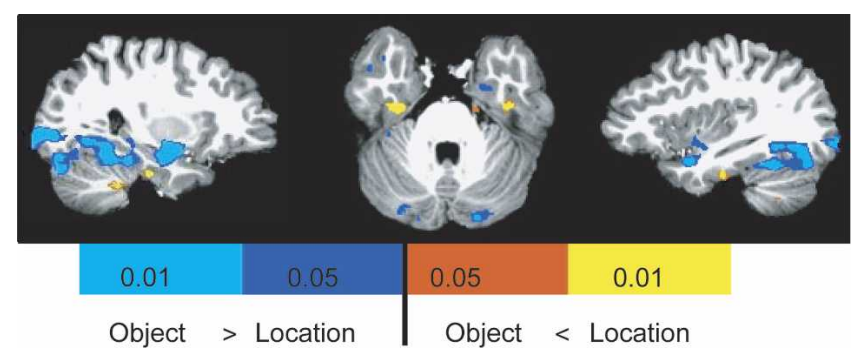

Figure 4. Task-specific encoding activity. Results from the randomeffect analysis across all 11 subjects showed an area of activation in the anterior parahippocampal cortex that was significantly greater for encoding during the Location task (warm colors) relative to the Object task (cool colors). Regions in the posterior ventral visual stream showed significant object encoding activity. The perirhinal cortex showed no taskspecific encoding activity. 


\section{Perirhinal Cortex}

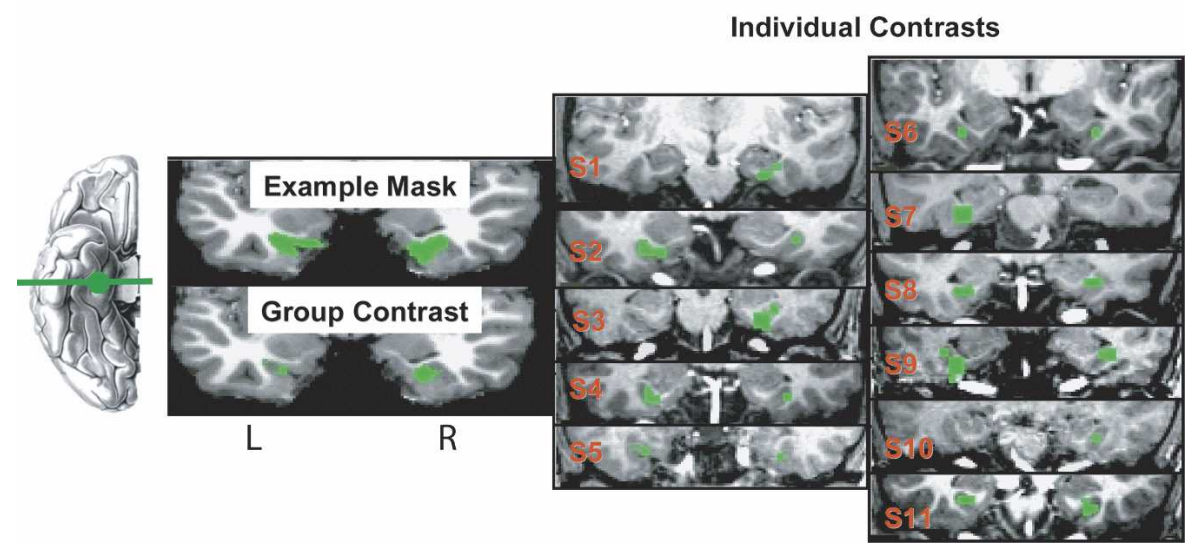

Figure 5. Encoding activity in the perirhinal cortex. Coronal sections are shown at the level of the posterior amygdala for a representative anatomical mask, the group effect, and the location of activity in each of the 11 individual subjects. For the group effect and across individual subjects, voxels are included that were significantly active during encoding of both tasks (green).

Location tasks, respectively. Moreover, across all 11 subjects, we found activity in the ventral occipitotemporal cortex that was specific for the Object task (see Fig. 4). If subjects were encoding object-location associations, one would expect to see similar activity in these areas for both tasks. Second, because of the type of stimuli used and the number and spacing of possible locations, these tasks were difficult for the subjects. The average performance was only $77 \%$ and $76 \%$ correct on the Object and Spatial tasks. This level of performance suggests that it is unlikely that subjects were encoding not just the critical task feature, but an additional association. Finally, from our post-session interviews with each subject, it was clear that the subjects did not appreciate the structure of the spatial layout. Not one subject was aware that the stimuli were presented on a $4 \times 4$ grid of 16 possible locations. Together, these data suggest that subjects were not encoding object-location associations, but were selectively encoding the stimulus domain cued in each task.

In sum, the present findings add to a growing body of literature indicating that the structures of the medial temporal lobe make distinct contributions to memory. Moreover, the unique activations of the medial temporal lobe cortical regions we observed were consistent with differential anatomical input to this region. Future studies combining careful anatomical segregation of the medial temporal lobe with hi-resolution scanning (e.g., Beauchamp et al. 2004) should provide further insight into the contribution of different medial temporal lobe structures to learning and remembering.

\section{Materials and Methods}

\section{Subjects and experimental tasks}

Eleven healthy subjects (seven male and four female) were recruited for functional imaging according to approved NIH protocols. All subjects gave informed consent.

During functional scanning, all subjects performed randomly interleaved blocks of the visual object and spatial recognition memory tasks (Fig.
2). For both tasks, the stimuli were trialunique, relatively nonverbalizeable, colorful patterns (Miyashita et al. 1991). These stimuli were chosen because amnesic patients with MTL damage are impaired at recognizing these stimuli with delays of 6 sec and longer, suggesting that these tasks rely on intact long-term memory systems (Buffalo et al. 1998). In the present study, the delays ranged from 18 to $33 \mathrm{sec}$. Using a button box, subjects performed an Old/New recognition judgment on either the stimulus (Object task) or the location of the stimulus on the screen (Spatial task). Through several pilot behavioral experiments, the Object and Spatial recognition memory tasks were equated for difficulty.

In the encoding phase of both tasks, the subjects were presented a series of six stimuli in varying locations on a computer screen. There were 16 possible stimulus locations. Each stimulus was presented for $2500 \mathrm{msec}$ with a 500msec interstimulus interval (ISI). Immediately preceding this phase, subjects were instructed either to "Memorize the Object" (visual Object task) or to "Memorize the Location" (Spatial task). Then, during a delay period, a scrambled image of a test stimulus was presented six times at the center of the screen at the same rate as the test stimuli $(2500 \mathrm{msec}, 500 \mathrm{msec}$ ISI). During this delay phase, subjects were instructed to "Watch the Picture." The same scrambled image was used throughout the experiment. Finally, during the recognition phase, six stimuli were again presented at the same rate $(2500 \mathrm{msec}, 500 \mathrm{msec}$ ISI). During the recognition phase of the visual Object task, three of the stimuli were repeated from the encoding phase, and three were new stimuli. Subjects were cued to "Recall the Object" and used a button box to make an Old/New recognition judgment. In the Object task, none of the six stimuli in the recognition phase was presented in a location that was used during the encoding phase of this block. During the recognition phase of the Spatial task, three of the stimuli were presented in the same location as a stimulus was presented during the encoding phase, and three were presented in new locations for this block. Subjects were cued to "Recall the Location" and used a button box to indicate recognition. All of the stimuli used in this phase were new

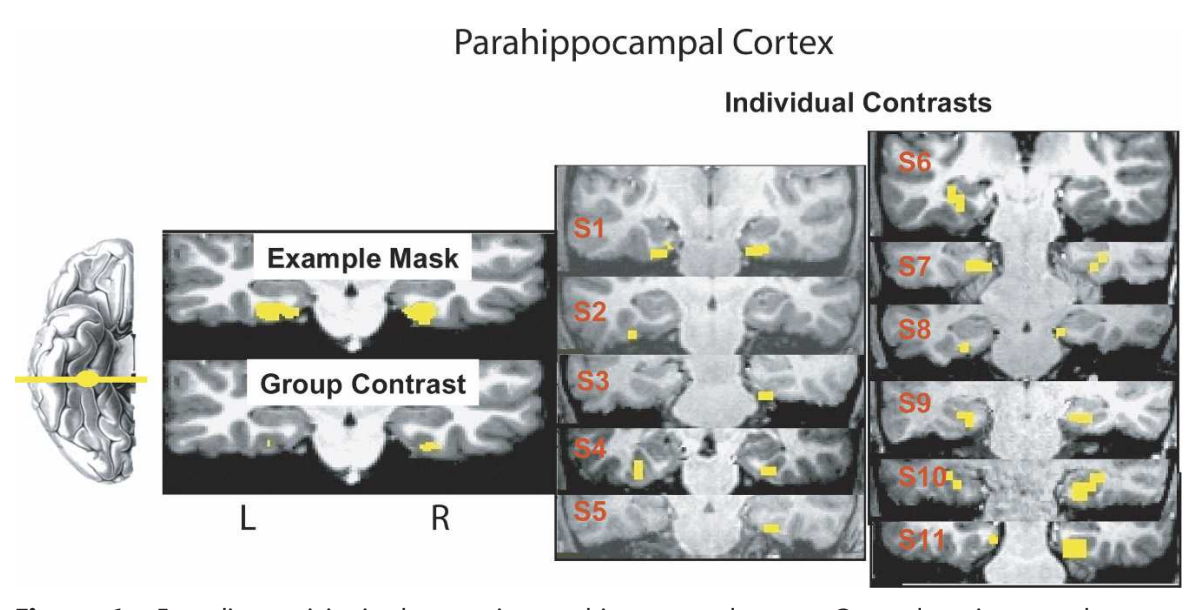

Figure 6. Encoding activity in the anterior parahippocampal cortex. Coronal sections are shown at the level of the anterior parahippocampal cortex for a representative anatomical mask, the group effect, and the location of activity in each of the 11 individual subjects. For the group effect and across individual subjects, voxels are included that were significantly active during location encoding relative to object encoding (yellow). 


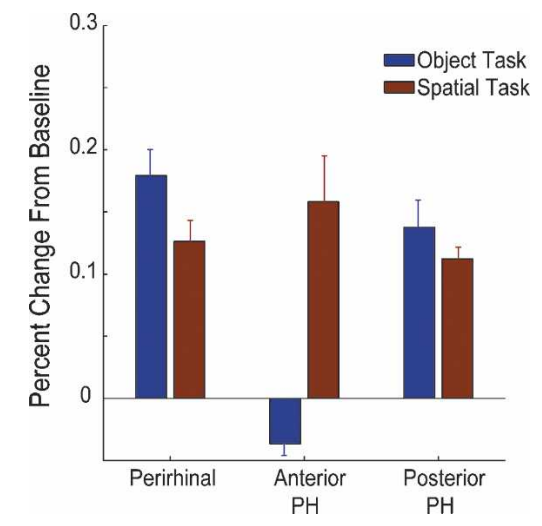

Figure 7. Average task activity. Averaged across all 11 subjects, the perirhinal cortex was active during the encoding phase of both the Object task (blue bar) and the Spatial task (red bar). In contrast, the anterior parahippocampal cortex was significantly more active during spatial encoding (red bar). The posterior parahippocampal cortex was active during both object and spatial encoding.

stimuli; only the locations of presentation were repeated. After the recognition phase and before the encoding phase of the next task block, subjects were cued to "Watch the Picture" and were shown six presentations of the scrambled image at the center of the screen. This post-recognition phase was used as the baseline in computing percent signal changes (see "Data Analysis" below).

Before scanning, all subjects were given a practice test to ensure that they understood all of the instructions and were able to perform the tasks. Subjects were explicitly told to pay attention only to the objects during the visual Object task and to the locations during the Spatial task. Subjects were also told that they would never see the same object presented in the same location, thus it would not be beneficial to try to memorize both the object and the location. To control for button pressing across the phases, during the encoding phase, the delay phase, and the post-recognition phase, subjects were instructed to press the center button on the button box when each stimulus appeared.

\section{Scanning parameters}

All scanning was performed on a General Electric 3 Tesla Signa scanner using a brain-specific quadrature head coil (Medical Advances). Higher-order shimming (Kim et al. 2002) was performed on the temporal lobes to minimize susceptibility artifacts arising from field homogeneities. Eight gradient-echo echoplanar functional scanning series using 164 -mm-thick axial slices covering the entire temporal lobe $(\mathrm{TE}=30 \mathrm{msec}$, $\mathrm{TR}=1500 \mathrm{msec}$, FOV $=240 \mathrm{~mm}$ ) were acquired with a $3.75 \times 3.75-\mathrm{mm}$ in-plane resolution. High-resolution anatomical Fast-SPGR images $($ FOV $=240 \mathrm{~mm}$, matrix $=256 \times 256,124$ slices, $1.2 \mathrm{~mm}$ thickness) were collected prior to the functional scans.

\section{Perirhinal and parahippocampal borders}

To localize the areas in the MTL that were active during the two tasks, we created an anatomical atlas that included the perirhinal cortex and the parahippocampal cortex for each individual subject according to the method described by Insausti et al. (1998). The perirhinal and parahippocampal cortices consist of tissue in the collateral sulcus bilaterally. Because the collateral sulcus has a highly variable shape and length (Insausti et al. 1995), we felt most confident about the cortical localization of the activations on individual brains, in the absence of spatial interpolation resulting from stereotaxic normalization. The landmarks we used for the borders of the perirhinal and parahippocampal cortices were as follows: The perirhinal cortex consists of tissue in both banks of the collateral sulcus, bilaterally, beginning $2 \mathrm{~mm}$ rostral to the level of the limen insula, or temporo-frontal junction. Although in primates, the more anterior temporopolar cortex is considered part of the perirhinal cortex (Suzuki and Amaral 1994b), we did not include this cortex in our mask of the perirhinal cortex because we did not obtain sufficient signal in this region in most of our subjects. The caudal limit of the perirhinal cortex was identified as $4 \mathrm{~mm}$ posterior to the level of the gyrus intralimbicus, or the caudal end of the uncus. At this level, the parahippocampal cortex replaces the perirhinal cortex in the collateral sulcus, and it replaces the entorhinal cortex medially. The caudal border of the parahippocampal cortex has not been as extensively characterized as the perirhinal cortex; accordingly, we considered the parahippocampal cortex to extend through the caudal extent of the hippocampus.

\section{Data analysis}

AFNI (Cox 1996; Cox and Hyde 1997) was used for removing the first two images of each functional series, motion correction (Cox and Jesmanowicz 1999), and statistical analyses. Following motion correction, all eight runs were concatenated prior to being submitted to a two-factor [TASK (Object or Spatial) and PHASE (encode/delay/recognition)] multiple regression resulting in a total of six regressors. Averaged time series were created by selectively averaging the time points during each of the three PHASE levels for both tasks. These time series data were then converted to percent change using the post-recognition phase as baseline and shifted by three images $(4.5 \mathrm{sec})$ to account for hemodynamic delay. The area under the averaged time series curve for each condition were submitted to a mixed effects three-factor ANOVA using subjects as a random factor and TASK and PHASE as fixed factors.

\section{Individual analyses}

Unbiased extraction of regionally specific averaged time series data were obtained using a combination of three masks applied to each individual's thresholded data. The first mask was an anatomical atlas of pre-specified regions of interest created on the high-resolution anatomical images. This anatomical mask was created using the landmarks for the borders of each region described above. The next mask was a whole-brain mask that eliminated voxels with an MR intensity less than the median image MR intensity value (see AFNI-3dAutomask). This mask was used to reduce the probability of including noisy voxels by eliminating non-brain voxels and voxels with insufficient signal to noise to detect small percent signal changes. The third mask was derived from the group ANOVA analysis by thresholding the Full Model F statistic $(F=5.42 ; P<0.00001)$ and the a priori comparison of means $t$-statistic $(P<0.05)$, that is, voxels that were significantly differentially active during the two tasks. The intersection of these three masks provided the data for the individual contrasts shown in Figures 5 and 6. For Figure 7, using the same masks, the average activity during encoding was calculated for each subject across each of the three regions.

\section{Group analysis}

For the group contrast data shown in Figures 4, 5, and 6, we combined the anatomically defined masks across subjects. We aligned the anatomical masks using AFNI's Talairach alignment, added the masks from all 11 subjects, and then created a combined mask for each anatomical region that included all voxels that were present in at least nine of the 11 subjects. This procedure was used to exclude effects due to extreme anatomical variability.

\section{Acknowledgments}

We are grateful to Ricardo Insausti for consultation on the identification of the borders of the perirhinal and parahippocampal cortices in MR images; to Monica Munoz-Lopez for helpful anatomical discussions; and to Robert Desimone, Mortimer Mishkin, and Leslie Ungerleider for comments on this manuscript.

\section{References}

Bar, M. and Aminoff, E. 2003. Cortical analysis of visual context. Neuron 38: $347-358$.

\section{Learning \& Memory}

www.learnmem.org 
Beauchamp, M.S., Argall, B.D., Bodurka, J., Duyn, J.H., and Martin, A. 2004. Unraveling multisensory integration: Patchy organization within human STS multisensory cortex. Nat. Neurosci. 7: 1190-1192.

Bohbot, V.D., Kalina, M., Stepankova, K., Spackova, N., Petrides, M., and Nadel, L. 1998. Spatial memory deficits in patients with lesions to the right hippocampus and to the right parahippocampal cortex. Neuropsychologia 36: 1217-1238.

Brewer, J.B., Zhao, Z., Desmond, J.E., Glover, G.H., and Gabrieli, J.D. 1998. Making memories: Brain activity that predicts how well visual experience will be remembered. Science 281: 1185-1187.

Buffalo, E.A., Reber, P.J., and Squire, L.R. 1998. The human perirhinal cortex and recognition memory. Hippocampus 8: 330-339.

Buffalo, E.A., Ramus, S.J., Clark, R.E., Teng, E., Squire, L.R., and Zola, S.M. 1999. Dissociation between the effects of damage to perirhinal cortex and area TE. Learn. Mem. 6: 572-599.

Cox, R.W. 1996. AFNI: Software for analysis and visualization of functional magnetic resonance neuroimages. Comput. Biomed. Res. 29: $162-173$.

Cox, R.W. and Hyde, J.S. 1997. Software tools for analysis and visualization of fMRI data. NMR Biomed. 10: 171-178.

Cox, R.W. and Jesmanowicz, A. 1999. Real-time 3D image registration for functional MRI. Magn. Reson. Med. 42: 1014-1018.

Davachi, L., Mitchell, J.P., and Wagner, A.D. 2003. Multiple routes to memory: Distinct medial temporal lobe processes build item and source memories. Proc. Natl. Acad. Sci. 100: 2157-2162.

Duzel, E., Habib, R., Rotte, M., Guderian, S., Tulving, E., and Heinze, H.J. 2003. Human hippocampal and parahippocampal activity during visual associative recognition memory for spatial and nonspatial stimulus configurations. J. Neurosci. 23: 9439-9444.

Epstein, R., Harris, A., Stanley, D., and Kanwisher, N. 1999. The parahippocampal place area: Recognition, navigation, or encoding? Neuron 23: $115-125$.

Insausti, R., Tunon, T., Sobreviela, T., Insausti, A.M., and Gonzalo, L.M 1995. The human entorhinal cortex: A cytoarchitectonic analysis. J. Comp. Neurol. 355: 171-198.

Insausti, R., Juottonen, K., Soininen, H., Insausti, A.M., Partanen, K., Vainio, P., Laakso, M.P., and Pitkanen, A. 1998. MR volumetric analysis of the human entorhinal, perirhinal, and temporopolar cortices. AJNR Am. J. Neuroradiol. 19: 659-671.

Jones, B. and Mishkin, M. 1972. Limbic lesions and the problem of stimulus-reinforcement associations. Exp. Neurol. 36: 362-377.

Kim, D.H., Adalsteinsson, E., Glover, G.H., and Spielman, D.M. 2002. Regularized higher-order in vivo shimming. Magn. Reson. Med. 48: $715-722$.

Kohler, S., Danckert, S., Gati, J.S., and Menon, R.S. 2005. Novelty responses to relational and non-relational information in the hippocampus and the parahippocampal region: A comparison based on event-related fMRI. Hippocampus 15: 763-774.

Mahut, H. 1971. Spatial and object reversal learning in monkeys with partial temporal lobe ablations. Neuropsychologia 9: 409-424.

Malkova, L. and Mishkin, M. 2003. One-trial memory for object-place associations after separate lesions of hippocampus and posterior parahippocampal region in the monkey. J. Neurosci. 23: 1956-1965.

Miyashita, Y., Higuchi, S., Sakai, K., and Masui, N. 1991. Generation of fractal patterns for probing the visual memory. Neurosci. Res. 12: 307-311.

Murray, E.A., Baxter, M.G., and Gaffan, D. 1998. Monkeys with rhinal cortex damage or neurotoxic hippocampal lesions are impaired on spatial scene learning and object reversals. Behav. Neurosci. 112: 1291-1303.

Pihlajamaki, M., Tanila, H., Hanninen, T., Kononen, M., Mikkonen, M., Jalkanen, V., Partanen, K., Aronen, H.J., and Soininen, H. 2003. Encoding of novel picture pairs activates the perirhinal cortex: An fMRI study. Hippocampus 13: 67-80.

Pihlajamaki, M., Tanila, H., Kononen, M., Hanninen, T., Hamalainen, A., Soininen, H., and Aronen, H.J. 2004. Visual presentation of novel objects and new spatial arrangements of objects differentially activates the medial temporal lobe subareas in humans. Eur. J. Neurosci. 19: 1939-1949.

Ploner, C.J., Gaymard, B.M., Rivaud-Pechoux, S., Baulac, M., Clemenceau, S., Samson, S., and Pierrot-Deseilligny, C. 2000. Lesions affecting the parahippocampal cortex yield spatial memory deficits in humans. Cereb. Cortex 10: 1211-1216.

Ranganath, C. and D'Esposito, M. 2001. Medial temporal lobe activity associated with active maintenance of novel information. Neuron 31: $865-873$.

Reber, P.J., Wong, E.C., and Buxton, R.B. 2002. Encoding activity in the medial temporal lobe examined with anatomically constrained fMRI analysis. Hippocampus 12: 363-376.

Rombouts, S.A., Barkhof, F., Witter, M.P., Machielsen, W.C., and Scheltens, P. 2001. Anterior medial temporal lobe activation during attempted retrieval of encoded visuospatial scenes: An event-related fMRI study. Neuroimage 14: 67-76.

Sommer, T., Rose, M., Glascher, J., Wolbers, T., and Buchel, C. 2005. Dissociable contributions within the medial temporal lobe to encoding of object-location associations. Learn. Mem. 12: 343-351.

Stark, C.E. and Squire, L.R. 2000. Functional magnetic resonance imaging (fMRI) activity in the hippocampal region during recognition memory. J. Neurosci. 20: 7776-7781.

Suzuki, W.A. and Amaral, D.G. 1994a. Perirhinal and parahippocampal cortices of the macaque monkey: Cortical afferents. J. Comp. Neurol. 350: $497-533$.

- 1994b. Topographic organization of the reciprocal connections between the monkey entorhinal cortex and the perirhinal and parahippocampal cortices. J. Neurosci. 14: 1856-1877.

Wagner, A.D., Schacter, D.L., Rotte, M., Koutstaal, W., Maril, A., Dale, A.M., Rosen, B.R., and Buckner, R.L. 1998. Building memories: Remembering and forgetting of verbal experiences as predicted by brain activity. Science 281: 1188-1191.

Received March 14, 2006; accepted in revised form June 29, 2006. 


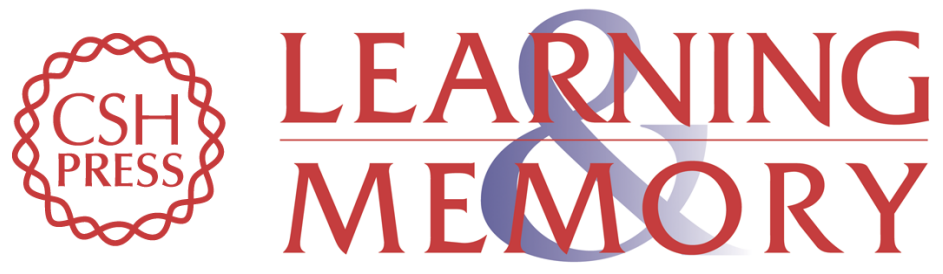

\section{Distinct roles for medial temporal lobe structures in memory for objects and their locations}

Elizabeth A. Buffalo, Patrick S.F. Bellgowan and Alex Martin

Learn. Mem. 2006, 13:

Access the most recent version at doi:10.1101//m.251906

References This article cites 32 articles, 9 of which can be accessed free at:

http://learnmem.cshlp.org/content/13/5/638.full.html\#ref-list-1

License

Email Alerting Receive free email alerts when new articles cite this article - sign up in the box at the Service top right corner of the article or click here. 